Technical Report

\title{
Ultrasound-Guided Interventional Release of Rotator Interval and Posteroinferior Capsule for Adhesive Capsulitis of the Shoulder Using a Specially Designed Needle
}

Kang Ahn, MD', Hyung-Joon Jhun, MD², Kyung-Man Choi, MD', and Yong-Seung Lee, MD'

From: ${ }^{1}$ Chronic Pain Management Center, Cha Biomedical Center, Kangnam Cha Hospital, Cha University, Seoul, and ${ }^{2}$ Center for Interventional Pain Management, Daejeon Woori Hospital, Daejeon, Republic of Korea

Address correspondence: Hyung-Joon Jhun, MD Center for Interventional Pain Management, Daejeon Woori Hospital 1419, Metrozone, Doonsan-dong Seo-gu, Daejeon, 302-531 Republic of Korea

E-mail: oemdoc21@gmail.com

Disclaimer: The first author, Kang Ahn, MD, developed the "curved Round Needle," the specially designed needle used in this study.

He possesses a Korean patent on

the needle. However, he has no stock or ownership in the company manufacturing the needle and has not been compensated for possessing

the patent by any individual or organization. None of the authors

receive reimbursements, fees, funding, or salary from any individual or organization related to the content of the manuscript.

Manuscript received: 05/16/2011 Revised manuscript received: 08/26/2011

Accepted for publication: 08/31/2011

Free full manuscript: www.painphysicianjournal.com
Background: Adhesive capsulitis is a common but poorly understood disorder of the shoulder. Various treatments have been developed to manage this condition, but the efficacy of these treatments is controversial. We developed an ultrasound-guided, minimally invasive interventional technique to manage adhesive capsulitis of the shoulder using a specially designed needle.

Objective: To describe the methodological approach of our intervention.

Study Design: Technical report.

Setting: Pain management clinic.

Methods: Thirteen participants with adhesive capsulitis of the shoulder underwent ultrasound-guided interventional release of the rotator interval and posteroinferior capsule using a specially designed curved Round Needle. A convex ultrasound probe was placed along the oblique coronal plane over the supraspinatus muscle and acromion. Under ultrasonographic guidance, the needle was passed through the supraspinatus muscle and superior labrum and reached the rotator interval. It was moved forward and backward until no resistance was felt. After finishing the rotator interval release, the needle was inserted toward the posteroinferior capsule. While the operator released the adhesion in the posteroinferior capsule by moving the needle forward and backward, an assistant gradually abducted the shoulder. When full abduction of the affected shoulder was achieved, the intervention was completed.

Results: Following our intervention, significant improvements in pain levels, glenohumeral range of motion, and Quick Disability of the Arm, Shoulder, and Hand scores were attained at follow-up.

Limitations: Technical report only. Efficacy of this technique is beyond the scope of the article.

Conclusion: Ultrasound-guided interventional release of the rotator interval and posteroinferior capsule appears to have clinical significance in the management of adhesive capsulitis of the shoulder.

Key words: adhesive capsulitis, shoulder, ultrasound, intervention, dry needling, technique

Pain Physician 2011; 14:531-537 
A dhesive capsulitis is a common but poorly understood disorder of the shoulder. Pain and generalized limitation of glenohumeral movement in both active and passive ranges of motion (ROMs) are characteristic findings of adhesive capsulitis of the shoulder (1). Its prevalence is estimated to be greater than $2 \%$ in the general population (2).

Most studies report a benign natural history of adhesive capsulitis; pain and limitation of motion usually last 2 to 3 years, and symptoms spontaneously resolve after this period (3). The external rotation ROM improves first, followed by abduction and internal rotation. Complete recovery, however, is infrequent. Approximately $7 \%$ to $15 \%$ of patients permanently lose their full ROM (4).

Various treatments have been developed to manage this condition, including oral corticosteroids, corticosteroid injections, physical therapy, nerve blocks, manipulation under anesthesia, distension arthrography, and arthroscopic or open release of contracture $(5,6)$. However, the efficacy of these treatments is controversial (7).

We developed an ultrasound-guided, minimally invasive interventional technique to manage adhesive capsulitis of the shoulder using a specially designed needle. This report describes the methodological approach of our intervention.

\section{Methods}

Thirteen patients ( 3 men, 10 women) with primary adhesive capsulitis of the shoulder who underwent an ultrasound-guided interventional dry needling technique in a chronic pain management clinic in Korea were recruited for this study. The ultrasound-guided interventional dry needling technique has been clinically applied in the clinic since early 2010 . The study participants were those who underwent the technique be- tween March and August 2010, with at least 6 months having elapsed since their last treatment.

The diagnosis of adhesive capsulitis in the participants was made by an expert in pain management. Diagnosis was based on the medical history and physical examination findings, such as pain in the shoulder and global loss of glenohumeral movement, with no underlying pathology, such as diabetes mellitus, previous upper extremity fracture, previous shoulder surgery, or stroke. All of the participants underwent ultrasonographic evaluation prior to the intervention by a physician experienced in musculoskeletal ultrasound using a high-frequency linear array transducer.

Participants were provided with comprehensive information on the benefits and potential risks (such as infection, bleeding, and post-needling soreness) of the intervention and gave informed consent prior to the treatment. Because this study was a follow-up of patients who had previously undergone the intervention, written informed consent was not required. The institutional review board approved the study protocol.

\section{Needle}

Fig. 1 shows the specially designed needle used in this technique: a curved Round Needle (Hansung Precision, Anyang, Korea). The needle was $0.9 \mathrm{~mm}$ in diameter and $120 \mathrm{~mm}$ long. A red circular sticker was attached to the triangular-shaped handle to indicate the side of the curved tip (Fig. 1A). It was streamlined, solid, and flexible, and it had a blunt, round, curved tip (Figs. $1 \mathrm{~B}$ and $\mathrm{C})$.

\section{Procedure}

The technique involved 2 release procedures: rotator interval and posteroinferior capsule release. The participant was asked to lie on a table in the prone position and was sedated with intravenous propofol

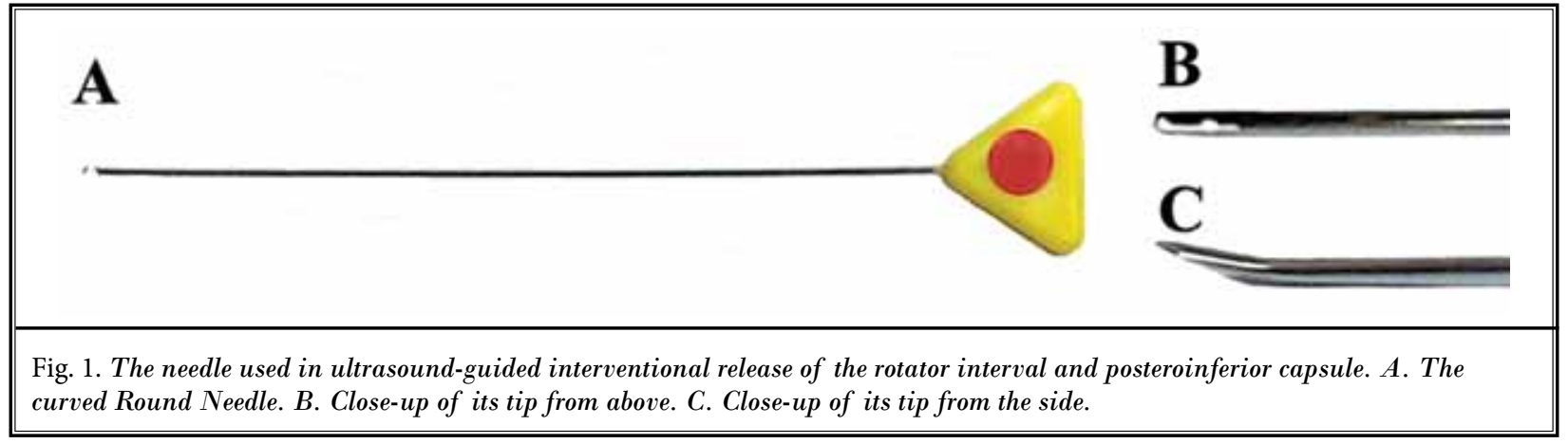


(Diprivan, $2-2.5 \mathrm{mg} / \mathrm{kg}$ for induction and $100-200 \mu \mathrm{g} /$ $\mathrm{kg} / \mathrm{min}$ for maintenance) to avoid pain during the procedure. A convex ultrasound probe was wrapped by a disposable protective cover. The cover was cleaned with a chlorhexidine-soaked cotton ball, which sterilized the cover and skin of the patient and transmitted ultrasound waves between them.

An assistant placed the probe along the oblique coronal plane over the supraspinatus muscle and acromion as the medial end of the acromion was spanned on the ultrasonic field. The medial side of the probe was pressed toward the acromion at an $80^{\circ}-85^{\circ}$ incline to the horizontal plane (Fig. 2A). In this view, the superior labrum was found on the lateral end of the supraspinous fossa beneath the supraspinatus muscle. A curved Round Needle was inserted at 2 fingers below and one finger medial to the lateral end of the scapular spine as the concave surface of the needle tip and red circular sticker faced upward (Fig. 2B). The needle was passed through the deltoid and supraspinatus muscles and was advanced 2-3 $\mathrm{mm}$ above the superior labrum. The needle was advanced another few millimeters until its tip reached the rotator interval. When it reached the target point, a cross-sectional image of the needle was shown as a hyperechoic spot located 2-3 $\mathrm{mm}$ above the superior labrum on the ultrasound screen (Fig. 2C). The needle was moved forward and backward, sliding on the surface of the superior labrum until no resistance was felt at the tip of the needle.
After finishing the rotator interval release procedure, the posteroinferior capsule release procedure was conducted. The convex surface of the probe was placed on the skin surface of the axillary recess, an inferior pouching of the joint capsule between the teres minor and subscapularis, and important structures, such as the humeral head, glenoid labrum, and infraspinous fossa, were identified (Fig. 3A). The curved Round Needle was inserted $2-3 \mathrm{~cm}$ cranial to the probe as the concave surface of the needle tip and the red circular sticker faced inferiorly (Fig. 3B). It was passed through the deltoid and infraspinatus and/or teres minor muscles and advanced obliquely toward the axillary recess. When it reached its target, the lower end of the needle was shown as a hyperechoic line on the ultrasound screen (Fig. 3C). The needle was moved forward and backward over the posteroinferior capsule. While the operator used the needle to release the capsular fibrosis under ultrasonographic guidance, an assistant gradually abducted the shoulder until no resistance was felt as determined by release of the capsular fibrosis. These release and passive abduction processes were performed simultaneously, and the degree of abduction increased gradually. When full abduction of the affected shoulder was achieved, the procedure was completed.

When biceps effusion was found, we injected 2-3 $\mathrm{mL}$ of diluted triamcinolone acetonide into the bicipital groove under ultrasonographic guidance. The so-

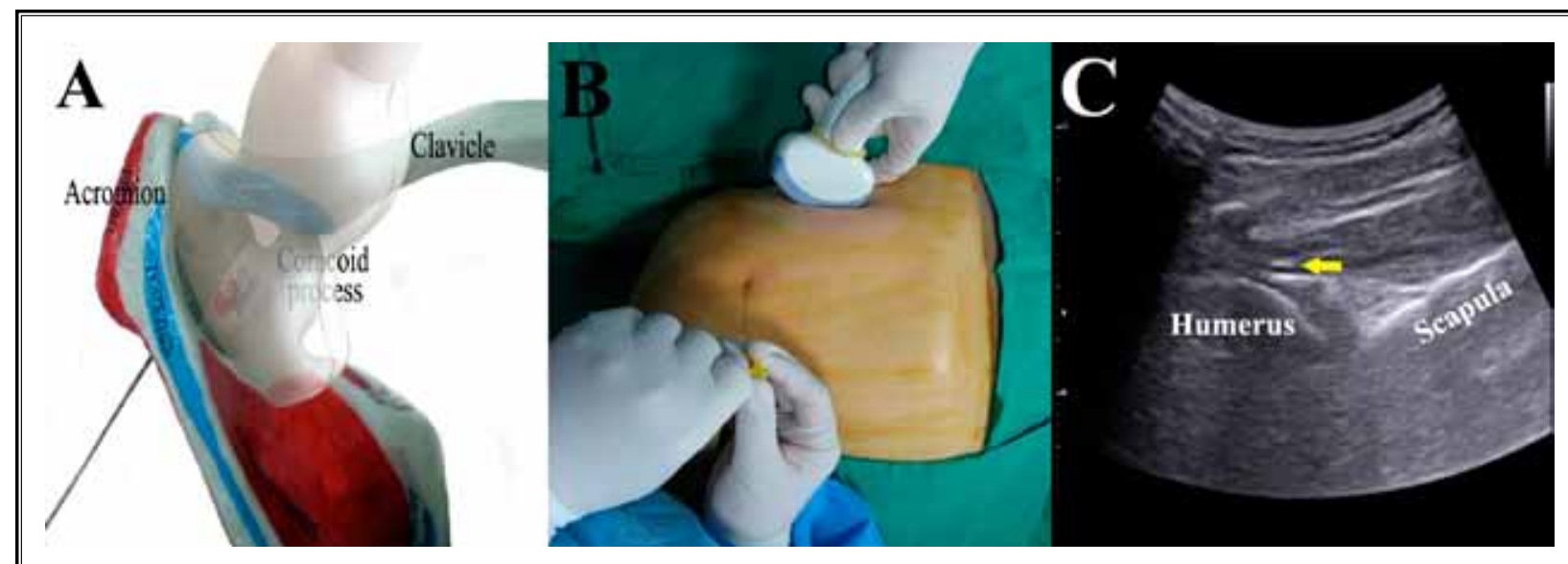

Fig. 2. The rotator interval release procedure in ultrasound-guided intervention for adhesive capsulitis of the shoulder. A. A curved Round Needle was advanced to the superior labrum and reached the rotator interval under ultrasonographic guidance in a human skeleton model. B. A curved Round Needle was inserted into a patient under ultrasonographic guidance. C. Ultrasound image of the rotator interval release procedure in a patient. The arrow indicates a cross-sectional image of the needle inserted at the superior labrum. 


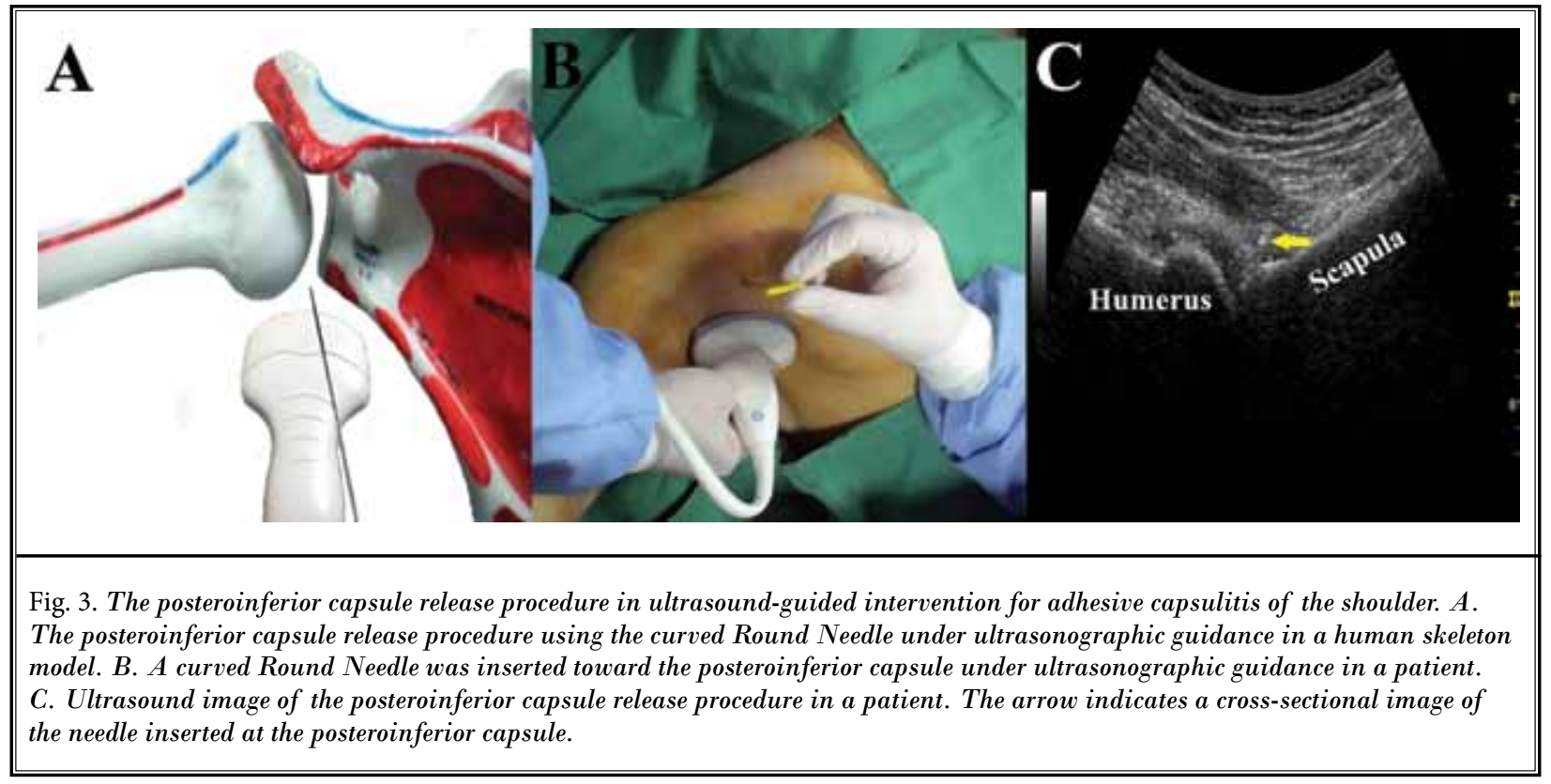

lution contained $4 \mathrm{mg}$ triamcinolone acetonide mixed with normal saline, which was one-tenth of the usual dose in steroid injection therapy. Based on our clinical experience, patients with adhesive capsulitis and comorbid biceps effusion tend to report persistent anterior shoulder pain, although their overall pain level and ROM improve after treatment. In this case, injection of triamcinolone acetonide into the bicipital groove helped to relieve anterior shoulder pain.

\section{Outcome measures}

We selected 3 outcome measures to evaluate the effectiveness of the treatment: self-rated overall pain score and pain level in specific situations, glenohumeral ROM of the affected shoulder, and the Quick Disability of the Arm, Shoulder, and Hand (QuickDASH) score.

We asked the participants to rate their overall pain level using a visual analog scale (VAS) that consisted of a $10 \mathrm{~cm}$ line anchored by 2 extremes. We also asked them to rate their pain level at night, at rest, and during daily activity using a VAS scale that consisted of a 10 $\mathrm{cm}$ line anchored by 2 extremes.

The QuickDASH, a shorter version of the DASH, consists of 2 components: the disability/symptom section and the optional high-performance sport/music or work modules. We used the disability/symptom section and did not use the optional modules in this study. The disability/symptom section of the QuickDASH consisted of 11 self-administered items that assessed physical function and symptoms of musculoskeletal disorders of the upper limb. The assigned values for all completed responses were simply summed and averaged. This value was then transformed to a score out of 100 by subtracting 1 and multiplying by 25: DASH score $=$ [(sum of the responses of individual items/number of completed responses) -1 1] $\times 25$. This transformation was performed to allow for easier comparison of the score with other measures on a 0 to 100 scale. A higher score indicates greater disability.

The pre-interventional baseline pain levels and QuickDASH scores were obtained using a self-administered questionnaire when the patients initially visited the hospital. Measurement of the pre-interventional baseline glenohumeral ROM and ultrasonographic evaluation of the affected shoulder were also conducted at the initial visit.

A follow-up evaluation of pain levels, QuickDASH score, and glenohumeral ROM was conducted at the hospital 3 weeks after the initial treatment. If a participant complained of limited improvement, the treatment was repeated at 3 week intervals.

The post-interventional pain scores and QuickDASH scores were obtained using a telephone survey. These outcome measures consisted of 15 items, which required 5-10 $\mathrm{min}$ to complete. To ensure the validity of the follow-up data, an independent researcher conducted the survey using a structured questionnaire in a standardized manner. 
We used the glenohumeral ROM measured at the participants' last visit as the post-interventional glenohumeral ROM in this study. The independent researcher also asked the participants whether their glenohumeral ROM had been preserved since their last visit when conducting the telephone survey.

The Wilcoxon signed rank test was used to evaluate the statistical significance of the differences between pre- and post-interventional outcome measures.

\section{RESULTS}

The average age of the participants was $56.8 \pm 11.1$ years. The average duration of pain prior to the treatment was $30.0 \pm 42.7$ months. Adhesive capsulitis affected the right shoulder in 8 participants $(61.5 \%)$ and the left shoulder in 5 participants (38.5\%). Physical therapy $(84.6 \%)$, medication $(84.6 \%)$, and acupuncture $(69.2 \%)$ were the 3 most frequently received previous treatments. Ultrasonography revealed that 12 participants $(92.3 \%)$ had a supraspinatus tendon tear, $3(23.1 \%)$ had a subscapularis tendon tear, 5 (38.5\%) had biceps effusion, and $7(53.8 \%)$ had biceps tendinitis.

Three $(23.1 \%)$ patients received the treatment once, $8(61.5 \%)$ underwent 2 interventions, and 2 $(15.4 \%)$ had 3 interventions. Of the 5 patients who were found to have biceps effusion in the ultrasonographic evaluation, 2 received one and 3 received 2 diluted triamcinolone acetonide injections into their bicipital groove. No significant or fatal adverse effect was reported following the treatment. The average followup period was $6.9 \pm 1.3$ months.

After the participants underwent ultrasound-guided interventional release of the rotator interval and posteroinferior capsule, the mean VAS pain score decreased by $6.9 \pm 2.2$ points (from $8.2 \pm 1.3$ at baseline to $1.2 \pm 1.5$ at follow-up; $P<0.01$ ). The VAS pain level at night decreased by $5.5 \pm 2.8$ points (from $6.3 \pm 3.0$ at baseline to $0.8 \pm 2.0$ at follow-up; $P<0.01$ ), that at rest decreased by $5.5 \pm 2.5$ points (from $6.3 \pm 2.6$ at baseline to $0.8 \pm 2.2$ at follow-up; $P<0.01$ ), and that during activity decreased by $5.2 \pm 2.7$ points (from $6.2 \pm 2.7$ at baseline to $1.1 \pm 1.9$ at follow-up; $P<0.01$ ). In terms of glenohumeral ROM, the angle of abduction increased by $94.2^{\circ} \pm 19.8^{\circ}$ (from $56.5^{\circ} \pm 12.7^{\circ}$ at baseline to 150.8 $\pm 17.5^{\circ}$ at follow-up; $P<0.01$ ), that of external rotation increased by $29.5^{\circ} \pm 8.3^{\circ}$ (from $26.3^{\circ} \pm 7.5^{\circ}$ at baseline to $55.8^{\circ} \pm 6.5^{\circ}$ at follow-up; $P<0.01$ ), and that of internal rotation increased by $29.0^{\circ} \pm 6.8^{\circ}$ (from $15.1^{\circ} \pm 5.0^{\circ}$ at baseline to $44.1^{\circ} \pm 4.5^{\circ}$ at follow-up; $P<0.01$ ). The QuickDASH score decreased by $46.2 \pm 14.6$ points (from
Table 1. Treatment responses of the patients $(n=13)$ with adhesive capsulitis of the shoulder to ultrasound-guided interventional release of the rotator interval and posteroinferior capsule.

\begin{tabular}{|c|c|c|c|}
\hline & Baseline & Follow-up & $\boldsymbol{P}$ \\
\hline \multicolumn{4}{|l|}{ Pain level (VAS) } \\
\hline Overall & $8.2 \pm 1.3$ & $1.2 \pm 1.5$ & 0.0002 \\
\hline Pain at night & $6.3 \pm 3.0$ & $0.8 \pm 2.0$ & 0.0005 \\
\hline Pain at rest & $6.3 \pm 2.6$ & $0.8 \pm 2.2$ & 0.0002 \\
\hline Pain on activity & $6.2 \pm 2.7$ & $1.1 \pm 1.9$ & 0.0002 \\
\hline \multicolumn{4}{|c|}{ Range of motion (degree) } \\
\hline Abduction & $56.5 \pm 12.7$ & $150.8 \pm 17.5$ & 0.0002 \\
\hline External rotation & $26.3 \pm 7.5$ & $55.8 \pm 6.5$ & 0.0002 \\
\hline Internal rotation & $15.1 \pm 5.0$ & $44.1 \pm 4.5$ & 0.0002 \\
\hline QuickDASH score & $56.1 \pm 13.8$ & $10.0 \pm 12.8$ & 0.0002 \\
\hline
\end{tabular}

VAS, visual analogue scale pain score;

DASH, disability of the arm, shoulder, and hand.

$56.1 \pm 13.8$ at baseline to $10.0 \pm 12.8$ at follow-up; $P<$ 0.01) (Table 1).

\section{Discussion}

After the participants underwent ultrasound-guided interventional release of the rotator interval and posteroinferior capsule, the outcome measures in this study improved significantly. Although we could not directly measure their glenohumeral ROM when we conducted the post-interventional follow-up study, all of the participants informed the independent researcher who conducted the telephone survey that their glenohumeral ROM had been preserved. Thus, we believe that our technique has clinical significance in the management of adhesive capsulitis of the shoulder.

Dry needling has been used in the management of musculoskeletal pain. Its effectiveness in the management of myofascial trigger points has been evaluated in randomized clinical trials and comprehensive reviews (8). Furthermore, dry needling has been used in the management of sophisticated musculoskeletal disorders, including tendinopathies, such as tennis elbow and patellar tendinosis $(9,10)$, and spinal stenosis through a transforaminal approach (11). It has also 
been used as an adjuvant treatment in musculoskeletal surgery (12).

In terms of the treatment mechanisms of our intervention, we hypothesize that our technique mechanically releases capsular adhesion of the shoulder and promotes the natural healing process, resulting in improvement in pain and ROM. Fibrosis and capsular contracture are important pathological findings in adhesive capsulitis $(13,14)$. Therefore, it is imperative that adhesions that affect the joint be released. We believe that our technique mechanically relieves adhesion and contracture in the shoulder capsule and surrounding soft tissues. Dry needling produces minute wounds that continuously generate a current of injury for several days or weeks in the lesion. Blood flow increases around the injured area. These processes promote the natural healing process and improve the underlying pathology in adhesive capsulitis of the shoulder.

Although we used a dry needle, our technique is unrelated to traditional Oriental (Chinese or Korean) medicine, including acupuncture. The morphology (shape, diameter, and length) and characteristics of the curved Round Needle are far different from those of an acupuncture needle. The diagnosis and treatment in this study were based on medical science, especially anatomy, and reported pathophysiological mechanisms of adhesive capsulitis of the shoulder.

We adopted posterior approaches to relieve adhesive capsulitis of the shoulder. To our knowledge, this is the first reported study to present a posterior approach to the rotator interval. Previous studies on shoulder joint injection or arthrography through the rotator interval presented an anterior approach for targeting the rotator interval $(15,16)$. The surgical instruments used in arthroscopic or open capsular release are introduced into the shoulder joint through an anterior approach (17). However, caution must be exercised to avoid damage to critical structures in the anterior shoulder, such as the subcoracoid bursa, subscapularis muscle and tendon, and superior glenohumeral ligament, when using an anterior approach (18). We believe that our posterior approach is safer than the anterior approach considering the anatomical structures in the posterior shoulder.

Our technique was developed as a technical advancement of a previously reported interventional dry needling technique for adhesive capsulitis of the shoulder (19). After introducing this technique, a skilled operator and assistant can perform a treatment in 20 minutes for a unilateral shoulder. Our technique is per- formed on an outpatient basis; the patient need not be hospitalized for this treatment. Characteristics of the specially made needle (streamlined shape, solid but flexible, and the round, blunt, curved tip) enable the operator to accurately target the lesion, reach a wide area, and allow for minute release of adhesions while minimizing unwanted tissue damage.

When we developed the previous technique, we took a magnetic resonance (MR) image to find any structural pathology that could cause the symptoms of adhesive capsulitis. We also evaluated the safety of the previous technique by comparing pre- and post-interventional MR images and found no structural damage resulting from the procedure (19). Our present technique is simpler than the previous one. Therefore, we concluded that MR scanning was not significantly useful considering its costs and benefits. Instead of MR scanning, all of the patients underwent ultrasonographic evaluation prior to intervention.

The participants in this study had neither a history of trauma nor a possible cause prior to the onset of symptoms, i.e., primary adhesive capsulitis. However, various types of shoulder pathologies, such as rotator cuff tears, biceps effusion, and biceps tendinitis, were found on ultrasonographic evaluation. Yoo et al (20) reported that $61.7 \%$ of patients with stage 2 idiopathic adhesive capsulitis showed some form of supraspinatus tendon pathology, most commonly partial-thickness tears, after evaluating MR arthrographic findings. Thus, we believe that detection of abnormal ultrasonographic findings in our participants was not unusual.

The design of this study, a technical report, poses a limitation, and further studies or randomized clinical trials are needed to evaluate the efficacy of our technique.

\section{Conclusions}

We developed an ultrasound-guided minimally invasive interventional technique to treat adhesive capsulitis of the shoulder. Thirteen participants with adhesive capsulitis underwent ultrasound-guided interventional release of the rotator interval and posteroinferior capsule using a specially designed curved Round Needle. An assistant placed a convex ultrasound probe along the oblique coronal plane over the supraspinatus muscle and acromion. Under ultrasonographic guidance, the needle reached the rotator interval and was moved forward and backward until no resistance was felt at the tip of the needle. After finishing the rotator interval release, the needle was inserted toward the pos- 
teroinferior capsule. While the operator released the adhesion in the posteroinferior capsule, an assistant gradually abducted the shoulder. When full abduction of the affected shoulder was achieved, this procedure was finished. Following our intervention, significant improvements in pain levels, glenohumeral ROM, and QuickDASH scores were obtained at follow-up. These results suggest that ultrasound-guided interventional release of the rotator interval and posteroinferior capsule have clinical significance in the management of adhesive capsulitis of the shoulder.

\section{ACKNOWLedgements}

The authors want to thank Ms. Ka-Yeon Lim for her help with the data collection.

\section{References}

1. Brue S, Valentin A, Forssblad M, Werner S, Mikkelsen C, Cerulli G. Idiopathic adhesive capsulitis of the shoulder: a review. Knee Surg Sports Traumatol Arthrosc 2007; 15:1048-1054.

2. Tighe CB, Oakley WS Jr. The prevalence of a diabetic condition and adhesive capsulitis of the shoulder. South Med J 2008; 101:591-595.

3. Reeves B. The natural history of the frozen shoulder syndrome. Scand J Rheumatol 1975; 4:193-196.

4. Siegel LB, Cohen NJ, Gall EP. Adhesive capsulitis: A sticky issue. Am Fam Physician 1999; 59:1843-1852.

5. Berghs BM, Sole-Molins X, Bunker TD. Arthroscopic release of adhesive capsulitis. J Shoulder Elbow Surg 2004; 13:180185 .

6. Harmon D, Hearty C. Ultrasound-guided suprascapular nerve block technique. Pain Physician 2007; 10:743-746.

7. Green S, Buchbinder R, Glazier R, Forbes A. Interventions for shoulder pain. Cochrane Database Syst Rev 2000. 2:CDool156.

8. Kalichman L, Vulfsons S. Dry needling in the management of musculoskeletal pain. J Am Board Fam Med 2010; 23:640646.
9. Connell DA, Ali KE, Ahmad M, Lambert $S$, Corbett S, Curtis M. Ultrasound-guided autologous blood injection for tennis elbow. Skeletal Radiol 2006; 35:371-377.

10. James SL, Ali K, Pocock C, Robertson C, Walter J, Bell J, Connell D. Ultrasound guided dry needling and autologous blood injection for patellar tendinosis. $\mathrm{Br}$ J Sports Med 2007; 41:518-521.

11. Ahn K, Jhun HJ, Lim TK, Lee YS. Fluoroscopically guided transforaminal epidural dry needling for lumbar spinal stenosis using a specially designed needle. BMC Musculoskelet Disord 2010; 11:180.

12. Bellemans J, Vandenneucker H, Van Lauwe J, Victor J. A new surgical technique for medial collateral ligament balancing: Multiple needle puncturing. J Arthroplasty 2010; 25:1151-1156.

13. Hannafin JA, Chiaia TA. Adhesive capsulitis. A treatment approach. Clin Orthop Relat Res 2000; 372:95-109.

14. Neviaser AS, Hannafin JA. Adhesive capsulitis: A review of current treatment. Am J Sports Med 2010; 38:2346-2356.

15. Dépelteau H, Bureau NJ, Cardinal E, Aubin B, Brassard P. Arthrography of the shoulder: A simple fluoroscopically guided approach for targeting the rotator cuff interval. Am J Roentgenol 2004; 182:329-332.

16. Souza PM, Aguiar RO, Marchiori E, Bardoe SA. Arthrography of the shoulder: A modified ultrasound guided technique of joint injection at the rotator interval. Eur J Radiol 2010; 74:e29-e32.

17. Warner JJ, Allen A, Marks PH, Wong P. Arthroscopic release for chronic, refractory adhesive capsulitis of the shoulder. J Bone Joint Surg Am 1996; 78:1808-1816.

18. Cheng PH, Modir JG, Kim HJ, Narouze S. Ultrasound-guided shoulder joint injections. Tech Reg Anesth Pain Manag 2009; 13:184-190.

19. Ahn K, Lee YJ, Kim EH, Yang SM, Lim TK, Kim YS, Jhun HJ. Interventional microadhesiolysis: A new nonsurgical release technique for adhesive capsulitis of the shoulder. BMC Musculoskelet Disord 2008; 9:12.

20. Yoo JC, Ahn JH, Lee YS, Koh KH. Magnetic resonance arthrographic findings of presumed stage-2 adhesive capsulitis: Focus on combined rotator cuff pathology. Orthopedics 2009; 32:22. 
